\title{
Die Digitalisierung von Personenbezogenen Dienstleistungen durch Online-Plattformen: Woher kommt die geringe Nutzung?
}

\author{
Pascal Mehrwald (iD · Frederik Heymann (D)
}

Eingegangen: 22. April 2020 / Angenommen: 12. Juni 2020 / Online publiziert: 30. Juni 2020

(C) Der/die Autor(en) 2020

Zusammenfassung Personenbezogene Dienstleistungen umfassen (alltags-)unterstützende Tätigkeiten beispielsweise im Bereich Haushalt, Kinderbetreuung oder Gartenarbeit und Umzüge. Diese Dienstleistungen werden allerdings von der Mehrheit der Deutschen noch nicht genutzt. Die Nutzungsraten sind gering. Obwohl es vielfältige Gründe für eine Nutzung gibt, insbesondere durch den Trend hin zu Doppelverdienerhaushalten, wird der Alltag nur selten durch die Inanspruchnahme personenbezogener Dienstleistungen unterstützt. Trotz einer starken Dynamik auf der Angebotsseite aufgrund neuer Online-Geschäftsmodelle scheinen Nutzungshürden vorhanden zu sein. Der Gegensatz von einem hohen Nutzungspotential und einer geringen tatsächlichen Nachfrage eröffnet die Möglichkeit einer Diskrepanz zwischen dem derzeitigen Dienstleistungsangebot und den Kundenbedürfnissen und -wünschen in Deutschland. Auf der Grundlage einer umfassenden Datenerhebung und Interviews mit Nutzern und Dienstleistungsplattformen präsentiert die vorliegende Arbeit 13 Annahmen, die für eine Nutzungssteigerung förderlich sein könnten. Zusätzlich soll diesem Forschungsbedarf durch eine repräsentative Befragung deutscher Haushalte begegnet werden, die sich zum aktuellen Zeitpunkt in der Erhebungsphase befindet. Die Daten ermöglichen einen Vergleich von Angebot und Nachfrage mit dem Ziel, Lücken und Verbesserungspotentiale aufzudecken. Die Ergebnisse werden zur bestehenden Literatur beitragen, indem sie Einblicke in die Kundenbedürfnisse geben und eine Analysebasis der neuen Online-Plattformen für Dienstleistungsanbieter liefern, die in der bestehenden Literatur unterrepräsentiert sind. Die Implikationen werden für Dienstleistungsanbieter, private Haushalte und die deutsche Wirtschaft von Interesse sein, da solche Dienstleistungen eine Mög-

\footnotetext{
P. Mehrwald $(\bowtie) \cdot$ F. Heymann

Technische Universität München, München, Deutschland

E-Mail: pascal.mehrwald@tum.de

F. Heymann

E-Mail: f.heymann@tum.de
} 
lichkeit darstellen, den Fachkräftemangel zu bekämpfen, die Gleichstellung der Geschlechter zu unterstützen und Arbeitsplätze für gering qualifizierte Arbeitnehmende zu schaffen.

Schlüsselwörter Personenbezogene Dienstleistungen · Personennahe Dienstleistungen · Online Dienstleistungsanbieter · Dienstleistungskunden · Personenbezogene Dienstleistungsanbieter · Nutzungshürden · Umfrage

\title{
The Digitalization of Personal Services Through Online Platforms: Where Does the Limited Usage Come from?
}

\begin{abstract}
Personal services support everyday activities such as household chores, childcare, gardening or relocating tasks. However, these services are not yet used by the majority of Germans. The usage rates are low. Although reasons for a usage are manifold, in particular due to the trend towards double-earning households, everyday life is rarely supported by personal services. Despite a strong dynamic on the supply side, caused by new online business models, usage hurdles seem to exist. The contrast between a high usage potential and a low actual demand creates the possibility of a discrepancy between the current offer of services and customer needs in Germany. Based on a comprehensive analysis of and interviews with users and service platforms, this paper presents 13 assumptions that could lead to an increased usage. In addition, this research need will be enriched by a representative survey of German households, which is still being conducted at the time of writing. The data allows for a comparison of supply and demand with the aim of identifying gaps and potential for improvement. The results will contribute to existing literature by providing insights into customer needs and lays the foundation for the analysis of new online service platforms as they are underrepresented in existing literature. The implications will be of interest to service providers, households and the German economy, because personal services on online-platforms are one way to address the shortage of skilled workers, support gender equality, and create jobs for lowqualified workers.
\end{abstract}

Keywords Personal services · Human services - Online service supplier - Service customers $\cdot$ Personal service suppliers · Usage hurdles · Survey

\section{Motivation, Relevanz und Forschungsfrage}

Die Digitalisierung und der damit verbundene Einsatz von innovativen, disruptiven Technologien ermöglicht es vielen Menschen derzeit bereits, Postpakete umzubuchen, Essen vom Sofa aus zu bestellen oder andere Dienste auf Abruf zu nutzen. Allerdings scheinen diese Möglichkeiten an eine Grenze zu stoßen, wenn es um personenbezogene Dienstleistungen geht. Personenbezogene Dienstleistungen werden an oder mit Menschen durchgeführt, sind alltagsunterstützend und sollen von oder mit einer vertrauenswürdigen Person erledigt werden (Robra-Bissantz et al. 2020; Lattemann et al. 2019). Zunehmend werden sie auch durch die Digitalisierung unter- 
stützt und verändert (Guerrero et al 2020). Hierzu gehören beispielsweise Friseure, Fahr- und Transportdienste, Kinder- und Tierbetreuung, Hilfe beim Umzug, der Autoreinigung, bei Abrechnungen oder der Planung einer privaten Feier. Darunter fallen auch haushaltsnahe Dienstleistungen, welche in der Literatur beschrieben werden, als Tätigkeiten, die sich noch fokussierter auf Tätigkeiten im Haushalt beziehen und normalerweise von Haushaltsmitgliedern oder Freunden ausgeübt werden, z. B. Unterstützung beim Putzen, Waschen, oder bei kleineren Reparaturen ohne dass spezielle Kenntnisse erforderlich sind. Sie erleichtern den Alltag und können auch von Fremden gegen Geld erfüllt werden (Reinecke et al. 2011). Im weiteren Verlauf wird nur der umfassendere Begriff der personenbezogenen Dienstleistungen verwendet. Das notwendige Vertrauen zwischen Dienstleistungsnehmenden und Dienstleistungsgebenden muss dabei geschaffen werden, damit sie gewillt sind, Transaktionen miteinander einzugehen (Hawlitschek et al. 2016). In Deutschland besteht ein hohes Marktpotenzial, da eine steigende Anzahl an Doppelverdienerhaushalten existiert (IfD Allensbach 2019). Darüber hinaus spielt der Wiedereintritt von Fachkräften in den Beruf oder die vollständige Vermeidung ihres Ausscheidens eine wichtige Rolle für die deutsche Wirtschaft (Reinecke et al. 2011).

Mögliche Gründe für die Inanspruchnahme von personenbezogenen Dienstleistungen gibt es viele, bspw. Zeitersparnis, Freizeit-(stress) oder mangelnde Fähigkeit, und trotzdem ist deren Nutzung in Deutschland immer noch gering. Nur $10 \%$ bis $18 \%$ der deutschen Haushalte beschäftigen beispielsweise derzeit eine Haushaltshilfe (Juncke et al. 2019), trotz eines geschätzten Potenzials von $40 \%$ (Bundesministerium für Wirtschaft und Energie o.J.).

Auf der Nachfrageseite von personenbezogenen Dienstleistungen sind deutsche Haushalte zunehmend durch Doppelverdienende gekennzeichnet (Ipsos 2013; Juncke et al. 2019). Während 2008 die Mehrheit der Deutschen noch bevorzugte, dass nur ein Elternteil berufstätig ist, befürwortete 2013 bereits der Großteil zwei berufstätige Partner/innen (Ipsos 2013). Heutzutage bedrängt vor allem Eltern das Gefühl von Zeitknappheit (McKinsey \& Company 2016). Hinzu kommt, dass die Arbeitszeiten der Deutschen oft über dem gewünschten Maß liegen (Weber und Zimmert 2018). In den letzten Jahrzehnten hat der globale Wohlstand erheblich zugenommen (Lange et al. 2018). Auch in Deutschland sind Einkommen und Vermögen in jüngster Zeit gestiegen (Deutsche Bundesbank 2019). Beispielsweise ist das durchschnittliche Nettovermögen deutscher Haushalte zwischen 2014 und 2017 um 9\% gestiegen (Deutsche Bundesbank 2019). Theoretisch würden steigende Einkommen den Arbeitnehmenden ermöglichen, Zeitknappheit auszugleichen, indem Freizeit durch den Kauf von personenbezogenen Dienstleistungen erworben wird. Beispielsweise die Auslagerung von ehemals selbst ausgeübter Hausarbeit an (semi-)professionelle Dienstleistende oder Laien könnte einen Zeitgewinn schaffen (Reinecke et al. 2011), der dann für den Beruf, die Familie oder andere Freizeitaktivitäten genutzt werden könnte. Der Kauf von Zeit kann sogar das Glücksgefühl steigern und den negativen Einfluss von Zeitdruck auf die Lebenszufriedenheit verringern (Whillans et al. 2017). Ebenfalls kann eine erhöhte Kaufkraft innerhalb der Gesamtgesellschaft erreicht werden und die stärkere Nachfrage nach personenbezogenen Dienstleistungen schafft Arbeitsplätze für Geringqualifizierte (Reinecke et al. 2011). Auch für die Gleichstellung der Geschlechter spielt die Thematik der personenbezogenen Dienst- 
leistungen eine entscheidende Rolle. Die Beschäftigungsquote von Frauen steigt; zwischen 2007 und 2017 ist die Erwerbstätigenquote von Frauen im Alter zwischen 20 und 64 um ca. 9\% gestiegen (Crößmann et al. 2018). Trotzdem ist es oft immer noch die Partnerin, die ihre Arbeitszeit reduziert, um Aufgaben im Haushalt zu übernehmen (Crößmann et al. 2018). Personenbezogene Dienstleistungen könnten u. a. Frauen (natürlich auch Männer und Diverse), die im Erwerbsleben bleiben oder wieder eine Beschäftigung aufnehmen wollen, unterstützen und ihre finanzielle Eigenständigkeit fördern (Reinecke et al. 2011).

Auf der Angebotsseite hat sich der deutsche Markt für personenbezogene Dienstleistungen zu einem bedeutenden Wirtschaftssektor entwickelt. Insbesondere die Verfügbarkeit von Dienstleistungen über neue Online-Plattformen hat den Markt in Bewegung gebracht (Bundesministerium für Wirtschaft und Energie o.J.). Im Vergleich zu den traditionellen Anbietern ist die Zahl der Online-Plattformen für personenbezogene Dienstleistungen seit 2014 enorm gewachsen (Bundesministerium für Wirtschaft und Energie o.J.). Trotzdem ist der Anteil der Deutschen, die ihre Erwerbstätigkeit reduzieren, um Zeit für die Pflege von Familienmitgliedern zu haben, in den letzten Jahren auf über ein Fünftel gestiegen; auch die Zahl der Deutschen, die ihre Erwerbstätigkeit ganz aufgeben, ist deutlich gestiegen (Die Bundesregierung der Bundesrepublik Deutschland 2016). Der prominenteste Grund für den Abbau von Beschäftigung ist die Kinderbetreuung. Bislang ist die Altenpflege nur ein untergeordneter Grund, Prognosen zeigen jedoch, dass dieser Trend in den kommenden Jahren aufgrund der alternden Gesellschaft noch an Bedeutung gewinnen wird (Die Bundesregierung der Bundesrepublik Deutschland 2016).

Obwohl also großes Nachfragepotenzial herrscht und ein breites Angebot an personenbezogenen Dienstleistungen besteht, werden diese Angebote nicht genutzt. Dieser Gegensatz wirft folgende Forschungsfrage auf:

Wie begründet sich die Diskrepanz zwischen dem verfügbaren (online) Angebot an personenbezogenen Dienstleistungen und den Bedürfnissen oder Wünschen der deutschen Nutzenden? Damit möchten wir folgende Beiträge zur Forschung und Praxis leisten: Ein Beitrag zur Forschung wird geleistet, indem zunächst eine repräsentative Datengrundlage geschaffen wird. Dies soll geschehen mittels einer repräsentativen Umfrage unter den (potenziellen) Nutzenden von personenbezogenen Dienstleistungen, dem Aufbau einer Datenbank, welche die aktuellen Anbietenden von entsprechenden Online-Plattformen im Detail analysiert, sowie unterstützenden Interviews mit Nutzenden und Plattformbetreibenden.

Aus dieser Datenbasis können (1) Meinungen zum Status-Quo erfasst werden (2) die Wünsche und Bedürfnisse der deutschen Nutzenden aufgenommen werden und (3) Diskrepanzen zwischen aktueller und potenzieller Nutzung identifiziert und quantifiziert werden. Daraus werden Empfehlungen abgeleitet, die eine höhere Nutzung und einen höheren Nutzen von personenbezogenen Dienstleistungen ermöglichen. Gleichzeitig können überprüfbare Annahmen gemacht werden, die das Nutzungsverhalten von Kunden und deren Bedürfnisse bei der Inanspruchnahme von Online-Plattformen für personenbezogene Dienstleistungen betreffen. Durch den stetigen Wandel von Internet, Kommunikationstechnologie und Kundenbedürfnissen sowie die damit verbundene Integration von Nutzenden, kann somit ein relevanter 
Beitrag für die Erforschung von Kundenbedürfnissen im Online-Plattformumfeld geschaffen werden; insbesondere für Plattformen von personenbezogenen Dienstleitungen.

Zum aktuellen Zeitpunkt befindet sich die erwähnte Umfrage in der Erhebungsphase. Jedoch sollen in diesem Beitrag 13 Annahmen vorgestellt werden, die aus den bisher gewonnen Daten und der Literatur stammen. Die Grundlage hierfür sind die genannte Datenbank mit analysierten Online-Plattformen sowie geführte Interviews mit Nutzenden und Plattformbetreibenden.

Unsere Beiträge für die Praxis ermöglichen Plattformbetreibenden und Dienstleistungsanbietenden, Angebote zu gestalten, die möglichst optimal auf Kundenbedürfnisse zugschnitten sind. Dadurch sind eine erhöhte Nutzung und ein verbesserter Nutzen zu erwarten. In Folge dessen wird erwartet, dass für Haushalte die Vereinbarkeit von Beruf und Familie oder Freizeit angenehmer und die Lebensfreude höher wird. Durch eine höhere Übereinstimmung von Angebot und Nachfrage kann auch eine Förderung der Geschlechtergleichstellung im Beruf und die Erwerbssicherung während der Kindererziehung unterstützt werden. Die genannte Unterstützung bei der Erwerbsteigerung oder -aufrechterhaltung in diversen Lebensphasen könnte ebenfalls ein Weg sein, dem Fachkräftemangel zu begegnen. Eine gesteigerte Erwerbstätigkeit ist entscheidend für die Wertschöpfung der deutschen Wirtschaft, insbesondere in Bezug auf den Fachkräftemangel. Darüber hinaus eröffnet ein verbessertes und dadurch wachsendes Angebot von personenbezogenen Dienstleistungen Verdienstmöglichkeiten für geringqualifizierte Arbeitskräfte.

\section{Hintergrund zu Dienstleistungen und Online-Plattformen}

Wir möchten zunächst auf Hintergründe zu Dienstleistungen und Online-Plattformen eingehen, die erarbeitet wurden. Hierbei sollen aktuelle nachfrageseitige Nutzungsfaktoren, -potentiale und -hürden dargestellt sowie auf die Angebotsseite, deren Herausforderungen und das Online-Angebot eingegangen werden.

\subsection{Aktuelle Nutzung}

Abhängig von der zugrundeliegenden Definition, liegt der Anteil an Haushalten, die personenbezogene Dienstleistungen nutzen, bei $10 \%$ bis $18 \%$ (Juncke et al. 2019) wobei ca. $80 \%$ der Haushaltshilfen illegal beschäftigt sind (Enste und Heldman 2017). Die Mehrheit der Nutzenden sind mit 75 \% kinderlose Haushalte (Juncke et al. 2019). Familien mit Kindern stellen, relativ zu ihrem Anteil an der Bevölkerung, viel seltener eine Haushaltshilfe ein (IFOK GmbH et al. 2014). Die Berücksichtigung des finanziellen Hintergrunds der Nutzenden macht deutlich, dass diejenigen, die personenbezogene Dienstleistungen in Anspruch nehmen, zu einer Gruppe mit hohem Einkommen gehören (Diener et al. 2015; Enste und Heldman 2017). Etwa jeder siebte Nutzende bucht personenbezogene Dienstleistungen online (Bitkom e. V. 2017). Vor allem Nutzende im Alter zwischen 30 und 49 Jahren nutzen die OnlineBuchungsfunktion; innerhalb dieser Gruppe hat jeder Fünfte die genutzten Dienstleistungen schon einmal online gebucht (Bitkom e. V. 2017). Generell können sich 
$42 \%$ der Deutschen vorstellen, eine Haushaltshilfe online zu buchen (Bitkom e. V. 2015). $29 \%$ sind daran interessiert, Kochdienstleistungen online zu buchen und $26 \%$ können sich vorstellen, Dienstleistungen für pflegebedürftige Angehörige online zu organisieren (Bitkom e. V. 2015). Bislang wird die Onlinebuchung am häufigsten für die Organisation von Nachhilfeunterricht genutzt, gefolgt von kleineren Reparaturen, Reinigungsdiensten und der Unterstützung von Angehörigen (Bitkom e. V. 2017). Auch McKinsey \& Company (2016) fanden heraus, dass die Befragten insbesondere für personenbezogene Dienstleistungen gerne digitale Technologien nutzen würden.

\subsection{Potentielle Nutzung}

Das Nachfragepotenzial wird auf insgesamt 16 Mio. Haushalte in Deutschland geschätzt (Bundesministerium für Wirtschaft und Energie 2015). Bei einer genaueren Betrachtung des Potenzials im Vergleich zur aktuellen Nachfrage sind noch Fragen offen. Es gibt beispielsweise auch eine potenzielle Nachfrage innerhalb junger Haushalte mit niedrigen bis mittleren Einkommen. Das steht im Gegensatz zur Mehrheit der aktuellen Nutzenden; Haushalte mit hohem Einkommen (Juncke et al. 2019). Wie das Interesse an personenbezogenen Dienstleistungen in Haushalten mit Kindern im Vergleich zu kinderlosen Haushalten ist, könnte auch Aufschlüsse liefern, wie das (online) Angebot an Dienstleistungen gestaltet sein müsste.

\subsection{Bisher identifizierte (offline) Nutzungshürden}

Rückschlüsse auf eventuelle Hürden für die Nutzung von Online-Plattformen von Dienstleistungen kann die Betrachtung bisheriger offline Nutzungshürden geben. Ein Faktor, der über die Inanspruchnahme von personenbezogenen Dienstleistungen entscheidet, ist der Preis (Eigenhüller 2019). Die Tatsache, dass solche Dienstleistungen auch aus eigener Kraft erbracht werden könnten, führt zu einer Nachfrage, die durch eine hohe Preissensibilität und Konkurrenz auf dem Schwarzmarkt gekennzeichnet ist (Bonin 2016). Für einige Deutsche, die versuchen, wieder ins Erwerbsleben einzusteigen, ist die Inanspruchnahme personenbezogener Dienstleistungen finanziell nicht tragbar (Reinecke et al. 2011; Wippermann 2011). Außerdem unterschätzen mögliche Nutzende den Nutzen dieser Dienstleistungen, während sie gleichzeitig die Kosten überschätzen; daher wird der bestehende Bedarf nicht in eine reale Nachfrage gewandelt (Reinecke et al. 2011). Der Markt für personenbezogene Dienstleistungen ist durch eine starke Intransparenz gekennzeichnet, was größere Anstrengungen bei der Suche nach dem richtigen Dienstleistungsanbietenden erfordert, was wiederum eine sinkende Nachfrage und Effizienz des Marktes zur Folge hat (Reinecke et al. 2011; Wippermann 2011). Auch Online-Buchungsplattformen funktionieren noch nicht zufriedenstellend und tragen daher weder wesentlich zur Erhöhung der Transparenz des deutschen Marktes für personenbezogene Dienstleistungen noch zur Senkung des administrativen Aufwandes bei (Bonin 2016). Selbst wenn die richtige dienstleistungsanbietende Person gefunden wird, hält der bürokratische Prozess der Buchung, Einstellung und Bezahlung einer legalen Haushaltshilfe die Nutzenden fern (IFOK GmbH et al. 2014; Juncke et al. 2019). 
Die Nutzung wird auch durch psychologische Gründe, Rollenvorstellungen (Reinecke et al. 2011) und gesellschaftliche Akzeptanz (Juncke et al. 2019) behindert. Personenbezogene Dienstleistungen haben in Deutschland ein Imageproblem (Bonin 2016). Zum einen gelten sie als Luxusgut, welches nur von Reichen genutzt wird (Bonin 2016), zum anderen wird ihnen nachgesagt, durch Schwarzarbeit erbracht zu werden (Reinecke et al. 2011). Deutsche geben an, ein schlechtes Gewissen zu haben, wenn sie die Aufgaben nicht selbst erledigen (Wippermann 2011). Dabei verzichten sie aus Zweifeln darüber, ob die Inanspruchnahme zu ihrem Selbstverständnis und sozialem Umfeld passt und fürchten um ihr Image als gute Eltern oder Hausfrau/-mann und ihrer Privatsphäre zu Hause (Wippermann 2011).

Ein Großteil der privaten Haushalte bezieht personenbezogene Dienstleistungen auf dem Schwarzmarkt (IFOK GmbH et al. 2014). Solange personenbezogene Dienstleistungen aus eigener Kraft erledigt oder zu einem niedrigen Preis auf dem Schwarzmarkt eingekauft werden können, wird es eine geringe Zahlungsbereitschaft geben. Damit können professionell Anbietende oder legale Anstellungen nicht konkurrieren. Dies wiederum stärkt den Schwarzmarkt (Juncke et al. 2019). Erschwerend kommt hinzu, dass der Schwarzmarkt nicht immer das gewünschte Qualitätsniveau liefern kann, was wiederum das Image der gesamten Branche durch schwer einschätzbaren Qualitätsstandards mindert (Juncke et al. 2019). Schließlich halten sich die Deutschen bei der Nutzung aufgrund von Sorgen vor geringer Qualität (Wippermann 2011) und mangelndem Vertrauen in Dienstleistende zurück (IFOK $\mathrm{GmbH}$ et al. 2014).

Wegen dieser Hürden schlägt sich die Nachfrage oft nicht in einer tatsächlichen Nutzung nieder. Frauen, die wieder in den Arbeitsmarkt eingetreten sind und personenbezogene Dienstleistungen in Anspruch nehmen, sehen diese als sehr vorteilhaft und entscheidend für eine dauerhafte Beschäftigung an (Wippermann 2011). Hinzu kommt, dass diejenigen Nutzenden, die bereits personenbezogene Dienstleistungen in Anspruch nehmen, die Nutzung eher ausweiten (Wippermann 2011). Zusammenfassend kann also bei der generellen Nutzung von solchen Dienstleistungen von einem Schwellenproblem gesprochen werden (Wippermann 2011), wobei die initialen Nutzungshürden maßgeblich sind. Ob darüber hinaus, bei der Online-Suche, Nutzung und Abwicklung entsprechender Dienstleistungen, weitere spezifische Nutzungshürden oder ein spezielles Verhalten der Nutzenden existieren, ist bisher nicht genauer untersucht worden.

\subsection{Dienstleistungsangebot und -markt in Deutschland}

Die Angebotsseite für Dienstleistungen gilt in Deutschland trotz aller bisheriger Aussagen als unterentwickelt (Juncke et al. 2019). Der Markt für personenbezogene Dienstleistungen ist, zumindest aus Sicht der Nachfrageseite, nicht optimal, da stark fragmentiert, kaum bedarfsorientiert oder nach Zielgruppen in Form von Angebot oder Qualität differenziert (Wippermann 2011). Die meisten Anbieter beschäftigen einen Mix aus Freiberuflern und sozialversicherungspflichtig Beschäftigten (Steiner et al. 2012). Hier sei nochmal an die hohe Quote an Schwarzarbeit erinnert. Die legale Erbringung von personenbezogenen Dienstleistungen ist durch einen hohen Anteil an geringfügig Beschäftigten gekennzeichnet, ca. ein Drittel ist als „Minijob“ 
beschäftigt (Becker et al. 2012). Die Branche ist geprägt von einem eher niedrigen Qualifikationsniveau, $31 \%$ der Beschäftigten der befragten Unternehmen haben keine abgeschlossene Berufsausbildung. Im Bereich der Reinigungsdienste haben fast $50 \%$ keine abgeschlossene Berufsausbildung (Becker et al. 2012). Dienstleistungsanbieter konzentrieren sich selten nur auf eine Kundenzielgruppe oder eine bestimmte Dienstleistung und streben vielmehr danach, eine breite Palette von Dienstleistungen anzubieten (Becker et al. 2012). Ihr Angebot reicht von der Betreuung von Kindern und Angehörigen über die Reinigung bis hin zum Kochen von Mahlzeiten. Dabei ist die Reinigung von Privatwohnungen meist das Kernprodukt (Becker et al. 2012).

Der Markt verzeichnet jedoch eine starke Dynamik. Innerhalb kurzer Zeit traten neue Anbieter, meist auf Basis von Online-Geschäftsmodellen, in den Markt ein, die den Wettbewerb stark verschärften (IFOK GmbH et al. 2014). Vor allem in Ballungsräumen drängen sich digitale Startups auf den Markt, die selbständige Dienstleistende an Kunden vermitteln (Enste und Heldman 2017). Die ehemals kleinen und lokal operierenden Unternehmen konkurrieren heute mit internationalen Akteuren, die oft über einen starken finanziellen Hintergrund verfügen, schneller auf Kundenwünsche reagieren, automatisierte Prozesse haben und somit ihre Leistungen zu niedrigeren Preisen anbieten können (IFOK GmbH et al. 2014; Enste und Heldman 2017). Diese international tätigen Startups verschärfen den Wettbewerb stark und können sich auf ihre enormen finanziellen Mittel stützen (Enste und Heldman 2017). Die Firma TaskRabbit beispielsweise, eine digitale Plattform für Hausund Heimwerkarbeiten mit Hauptsitz in den USA, ist seit 2019 auf dem deutschen Markt tätig (Presseportal 2019). Vorher wurden Finanzierungen in Höhe von insgesamt 38 Mio. USD eingesammelt und zuletzt wurde das Unternehmen von IKEA gekauft (Dickey 2017).

\subsection{Herausforderungen auf Seiten des Angebots}

Die Diskrepanz zwischen den Preisen, die für eine legal beschäftigte Dienstleistungserbringung benötigt werden, um z. B. die Kosten für die Sozialversicherung der Beschäftigten zu decken, und der Zahlungsbereitschaft bzw. -fähigkeit potenzieller Kunden ist eine große Herausforderung für Dienstleistungsunternehmen (Eigenhüller 2019). Die Anbietenden konkurrieren nicht nur untereinander, was bei steigender Nachfrage zu höheren Preisen führen würde, sondern auch mit dem Schwarzmarkt (Becker et al. 2012). Ein weiteres Problem für Dienstleistungsfirmen ist es, vertrauenswürdiges und qualifiziertes Servicepersonal am richtigen Standort zu finden (Juncke et al. 2019). Zuverlässige Mitarbeitende zu rekrutieren, ist ein wesentlicher Erfolgsfaktor für Dienstleistungsunternehmen (Steiner et al. 2012). Allerdings ist die individuelle Qualifikation potenzieller Mitarbeitende schwierig zu bewerten, da es in der Branche keine formalen Abschlüsse gibt, die speziell für personenbezogene Dienstleistungstätigkeiten qualifizieren (Juncke et al. 2019). Diese Probleme werden durch das schlechte Image der Branche noch verstärkt, da die Tätigkeiten bekanntermaßen körperlich anstrengend und in der Regel unattraktiv sind (Becker et al. 2012). Daher verringert das schlechte Image der Branche nicht nur die Nachfrage, sondern auch das Angebot (Juncke et al. 2019). 
Für Dienstleistungsanbieter ist neben der Gewinnung von Neukunden auch die Kundenbindung entscheidend (IFOK GmbH et al. 2014). Essentiell für die Zufriedenheit und Bindung von Kunden ist die Qualität der Dienstleistungen, die auch ein Differenzierungsmerkmal gegenüber dem Schwarzmarkt darstellt, das es ermöglicht, Marktanteile zu gewinnen und Nichtnutzende zu überzeugen (IFOK GmbH et al. 2014).

\subsection{Online-Angebot}

Neben einer wachsenden Zahl klassischer Dienstleistungsanbieter ist in den letzten Jahren auch die Zahl der Online-Plattformen für personenbezogene Dienstleistungen stark gewachsen. Besonders häufig werden über die Online-Plattformen Reinigungsdienste, aber auch Dienstleistungen für Möbelbau-, Maler- und Gartenarbeiten angeboten (Enste und Heldman 2017).

Die vermeintlichen Vorteile dieser Online-Angebote sind breit gefächert: Die Suche wird erleichtert und die Dienstleistungsanbieter garantieren oft hohe Qualitätsstandards, die durch Bewertungen anderer Kunden überprüft werden können (Bitkom e. V. 2017). Darüber hinaus tragen die Online-Plattformen dazu bei, die Arbeitsauslastung der Dienstleistungsanbietenden zu erhöhen und ihre Abhängigkeit von einzelnen Kunden zu verringern (Bitkom e. V. 2017). Somit wird das Anbieten einer Tätigkeit für Gelegenheitsjobber als auch für diejenigen interessant, die dort ihrer Haupterwerbstätigkeit nachgehen.

Des Weiteren könnten diese Online-Datenbanken ein Instrument sein, um die Transparenz des Marktes zu erhöhen und sein Wachstum zu stimulieren (Steiner et al. 2012). Mit einer massiven Werbepräsenz bieten Online-Plattformen ihre Dienstleistungen insbesondere in deutschen Großstädten an (IFOK GmbH et al. 2014). Die Buchung von Dienstleistungen über Online-Plattformen ist sehr einfach und schnell möglich (Bundesministerium für Wirtschaft und Energie 2015). Ein generelles Problem der Plattformen ist allerdings die Diskrepanz zwischen beworbenem, realem und angenommenem Angebot sowie Unsicherheiten bezüglich Gewährleistungen und Steuerabzügen (Bundesministerium für Wirtschaft und Energie 2015).

Nielen et al. (2017) analysierten Unternehmen im Raum Düsseldorf und stellten fest, dass in der Branche der personenbezogenen Dienstleistungen ein Fünftel des Umsatzes online erwirtschaftet wird. Über $96 \%$ der Unternehmen für personenbezogene Dienstleistungen in diesem Bereich berichten von einem zunehmenden Wettbewerb innerhalb der letzten fünf Jahre (Nielen et al. 2017). Einer der Faktoren, die den Wettbewerb verstärken, ist ein zunehmendes Angebot von OnlineDienstleistungen (Nielen et al. 2017). Gleichzeitig ist auch fraglich, wie sich Nutzende eine technologische Einbindung in den gesamten Nutzungsprozess von personenbezogenen Dienstleistungen vorstellen. Wie schaffen es Online-Plattformen, das notwendige Vertrauen und bürokratische Unterstützung zu gewährleisten?

Der Markt der personenbezogenen Dienstleistungen ist durch eine Vielzahl von Geschäftsmodellen gekennzeichnet (IFOK GmbH et al. 2014). Seit Anfang 2014 gibt es nun eine wachsende Zahl von Online-Geschäftsmodellen, die sich in zwei Gruppen unterteilen lassen: Vermittlungsplattformen und Online-Marktplätze (IFOK $\mathrm{GmbH}$ et al. 2014). Diese Geschäftsmodelle unterscheiden sich vor allem nach 
der Art und Weise, wie das Personal des Dienstleisters beschäftigt wird. Während Vermittlungsagenturen und -plattformen nur selbständige Anbieter an Kunden vermitteln und einen geringen Einfluss auf die daraus resultierenden Verträge haben, haben Dienstleistungsagenturen Personal angestellt und schließen Verträge direkt mit den Kunden ab (IFOK GmbH et al. 2014). Wie das optimale Geschäftsmodell, für die Plattform, sowie Dienstleistungsanbietende und -nehmende aussieht, ist auch eine erfolgskritische Frage, um personenbezogene Dienstleistungen mittels OnlinePlattformen zu etablieren und die Nutzung sowie den Nutzen zu steigern.

\section{Methode}

Um die aufgestellte Forschungsfrage nach der aktuellen Diskrepanz zwischen verfügbarem Online-Angebot und den Wünschen sowie Bedürfnissen der potenziellen Nutzenden von personenbezogenen Dienstleistungen umfassend zu untersuchen, wird eine repräsentative Datenerhebung durchgeführt. Diese erfolgt im Rahmen des Forschungsprojektes KUSTOMA (Förderkennzeichen 02K17A030). Die Datenerhebung umfasst dabei eine repräsentative Umfrage unter (potentieller) Nutzenden, eine Erhebung von Unternehmensdaten von Online-Plattformen personenbezogener Dienstleistungen, sowie Interviews mit aktuellen Nutzenden und Plattformbetreibenden. Durch diese Triangulation (Thurmond 2001) könnte den Defiziten von nur einer Datenquelle entgegengewirkt werden, was insbesondere bei dieser komplexen Fragestellung als sinnvoll anzusehen ist. Zudem erscheint die Kombination der drei Erhebungsquellen zielführend, um die möglichen Diskrepanzen aufzudecken. Es werden alle drei Erhebungsmethoden vorgestellt. Bisher sind die Analyse der Plattformen sowie die Interviews bereits abgeschlossen.

\subsection{Repräsentative Umfrage unter potentiellen Nutzenden}

Im Rahmen der Forschungsarbeit wird eine Umfrage durchgeführt, die die Kundenwünsche und -bedürfnisse von (potentiellen) Nutzenden von personenbezogenen Dienstleistungen in Deutschland abfragen soll. Für die Umfrage wird ein repräsentatives Sample für Deutschland befragt, sodass ca. 1000 potenzielle Haushalte teilnehmen werden.

Die Umfrage hat dabei drei Ziele. Zunächst sollen die aktuelle Nutzung und Nachfrage von personenbezogenen Dienstleistungen, sowie positive und negative Erfahrungen der Nutzenden in Deutschland erfasst werden. Des Weiteren sollen die Wünsche und Bedürfnisse der (potentiellen) deutschen Kunden abgefragt werden. Als drittes Ziel sollen Einblicke in die Optimalvorstellung der Nutzung gewonnen werden. Darüber hinaus werden verschiedene wissenschaftlich validierte Konstrukte abgefragt, um die Einflüsse von technologischer Affinität oder Bedenken hinsichtlich der Privatsphäre im online und peer-to-peer Kontext zu untersuchen. Um den aktuellen Nutzungshürden zu begegnen, sollen zudem Einstellungen zu verschiedenen Technologien (bspw. GPS-Tracking) zur Prozessunterstützung, als auch Erfahrungen und Wünsche zu vertrauensbildenden Maßnahmen hinterfragt werden. Zusätzlich werden auch gewünschte Zahlungsmodelle im Bereich der personenbe- 
zogenen Dienstleistungen abgefragt. Der thematische Bereich der Kinderbetreuung wird vertieft untersucht, da diese die vertrauensintensivste Dienstleistung darstellt. Die Ergebnisse der Umfrage liefern somit wesentliche Erkenntnisse, um aktuelle Diskrepanzen zu identifizieren und Annahmen treffen zu können, wie technologieunterstützt die Nutzung und der Nutzen von personenbezogenen Dienstleistungen erhöht werden kann. Die Umfrage wird von einem professionellen Umfrageunternehmen erhoben und online durchgeführt. Teilnehmer der Umfrage werden durch das Umfrageinstitut durch monetäre Anreize zur Teilnahme motiviert.

\subsection{Unternehmenserhebung zu Anbietern personenbezogener Dienstleistungen}

In der bereits abgeschlossenen Erhebung zu Anbietern personenbezogener Dienstleistungen wurden 215 Anbieter identifiziert und schließlich 175 anhand von Sekundärdaten analysiert (145 deutschsprachige Unternehmen, davon 135 aus Deutschland, 30 aus den USA). Nicht mehr aktive Unternehmen oder nicht nutzbare Internetseiten wurden ausgeschlossen.

Für die Analyse wurden die Internetseiten analysiert, Anmeldungen auf den Seiten durchgeführt und, wo möglich, Rezensionen gelesen und mit der Seite interagiert oder auch Unternehmen angerufen. Die Analyse umfasste, neben Basisinformationen über die Unternehmen, die von den Unternehmen abgedeckten Dienstleistungen, primäre Prozessschritte (wie z. B. Suche, Vermittlung, Durchführung, Abwicklung) und sekundäre Prozessschritte (wie z.B. die administrative Unterstützung zu Steuerfragen, Versicherung und Dokumentation), vertrauensbildende Maßnahmen, Qualitätssicherungsmaßnahmen und deren Geschäftsmodelle ${ }^{1}$.

Zur Sicherstellung der Reliabilität wurde eine Forschungsgruppe „Uberize your daily life" an der Technischen Universität München gegründet, welche sich mit der Analyse befasste. Mitglieder der Forschungsgruppe waren Mitarbeitende des Lehrstuhls für Strategie und Organisation sowie Studierende. Die Unternehmen wurden nach der Art der Dienstleistungen, die sie anbieten, kategorisiert. Im finalen Sample waren Dienstleistungsanbieter aus dem Bereich Botengänge und Fahrdienstleistungen, Kinderbetreuung, Organisation des eigenen Privatlebens, Reparaturen und Pflege rund um den Haushalt und das Auto, Tierbetreuung sowie sonstige Dienstleistungen enthalten. Ein vorläufiges Ergebnis ist beispielsweise, dass meist nur die Abwicklung und die Suche im Gesamtprozess unterstützt werden, sowie viele Unternehmen eher klein sind. Die Technologieunterstützung ist allgemein als niedrig einzuschätzen.

\subsection{Interviews mit Nutzenden und Plattformbetreibenden}

Anhand von Interviews mit Nutzenden der Angebots- und Nachfrageseite, wurden mögliche Wünsche und Bedürfnisse identifiziert, die auch in der beschriebenen Umfrage berücksichtigt sind und gefestigt werden sollen. Die literaturbasierten Semistrukturierten Interviews wurden mit insgesamt 27 Personen geführt; davon waren 13 Personen (potenziell) Nutzende als Nachfrager und Anbieter, sowie 14 Personen

\footnotetext{
1 Geschäftsmodelle konnten im Detail von 124 operativ tätigen Anbietern analysiert werden.
} 
Mitarbeitende von Online-Plattformen und kinderbetreuungsanbietenden Unternehmen. Befragte Personen standen in Verbindung mit unter anderem folgenden (teils mehreren) Unternehmen: TaskRabbit, Betreut.de, Die Notfallmamas, HalloBabysitter.de, Stressfrei, Jeffrey GmbH, Helpling. Themenschwerpunkte der Semi-Strukturierten Interviews waren Wechselkosten und Kundenbindungsmaßnahmen, persönliche, administrative, technologische Nutzungshürden und Erfahrungen. Die Interviews wurden transkribiert, strukturiert inhaltlich codiert und analysiert (Kuckartz 2016). Hieraus wurden Inhalte für Anforderungskataloge an eine Online-Plattform abgeleitet.

Gewünscht wurde beispielsweise eine sehr detaillierte Aufgabenbeschreibung, eine beidseitige Bewertungsmöglichkeit und Historie oder Fotodokumentationen, um Vertrauen in Online-Dienstleistungsplattformen und die Nutzung zu fördern. Plattformbetreibende beschrieben das Problem der Kundenabwanderung, wobei hier die finanziellen Abwanderungshürden nicht ausreichend seien und Kunden vermutlich ein Gemeinschaftsgefühl und andere Mehrwerte durch eine Plattformnutzung erwarten. Durch die weitere Auswertung der Interviews und Integration in die Gesamtdatenbasis im zukünftigen Verlauf werden vielversprechende Erkenntnisse erwartet, die der Erhöhung der Nutzung von Online-Plattformen für Dienstleistungen fördern können.

\section{Momentane Annahmen \& Ausblick}

Aus den bisherigen Erkenntnissen der Literatur, und den bisher verfügbaren Daten aus Datenbank und Interviews, können bereits folgende Annahmen abgeleitet werden, die für eine Nutzungssteigerung von personenbezogenen Dienstleistungen förderlich sein könnten:

1. Für eine Nutzungssteigerung sollte die Vermittlung von Dienstleistungen ausgebaut und professionalisiert werden, um den Zeitaufwand für die Suche nach einem passenden Dienstleistungsanbietenden zu reduzieren und eine hohe Qualität zu gewährleisten. Hierbei scheinen Online-Plattformen der richtige Weg.

2. Das Vertrauen der Nutzenden in die Qualität der Dienstleistungen und die Professionalität der Ausführung ist weiterhin entscheidend und ausschlaggebend für oder gegen eine Inanspruchnahme von Dienstleistungen.

3. Der Preis allein entscheidet nicht über die Zahlungsbereitschaft für oder Nutzung von personenbezogenen Dienstleistungen.

4. Nutzende präferieren eine mobile Applikation gegenüber einer reinen Nutzung über eine Internetseite.

5. Nutzende sind bereit, mehr Informationen zu teilen, als angenommen wird oder als derzeit möglich ist, um Vertrauen unter Nutzenden zu erhöhen.

6. Legal operierende Dienstleistungsunternehmen haben die Chance, Marktanteile zu gewinnen, indem sich ihr Angebot von dem auf dem Schwarzmarkt abhebt, was insbesondere im Hinblick auf das Qualitätsniveau, Vertrauen sowie Bürokratieunterstützung möglich ist. 
7. Die Branche der personenbezogenen Dienstleistungen bedarf einer Imagekampagne in Deutschland. So könnte eine gesteigerte Wertschätzung und Akzeptanz in der breiten Gesellschaft erreicht und psychologische Hürden aufgrund von traditionellen Rollenmodellen überwunden werden.

8. Strategisch wertvoll könnte es sein, den Nutzungshürden innerhalb der Mittelschicht entgegenzuwirken und die entlastende Wirkung, die persönliche und soziale Attraktivität sowie die Normalität solcher Dienstleistungen zu betonen (Wippermann 2011). Ähnliches gilt für die Gruppe der jungen Leistungserbringenden, die stark wachsen wird und eine Vorbildfunktion hat, die andere Gruppen beeinflusst (Wippermann 2011).

9. Gezielte finanzielle Unterstützung der Nachfrageseite, durch bspw. erweiterte Berücksichtigung bei Steuerzahlungen, kann eine erfolgreiche Methode sein, die geringe Zahlungsbereitschaft der Nutzenden zu erhöhen, um höhere Preise für legale Dienstleistungen und eine geringere Attraktivität des Schwarzmarktes zu ermöglichen (Juncke et al. 2019).

10. Die Unterschätzung des Nutzens und gleichzeitige Überschätzung der Kosten muss in der Wahrnehmung der Nutzenden umgekehrt werden.

11. Der administrative Aufwand und die technologische Unterstützung muss so in die Online-Prozesse und Technologie integriert sein, dass die Nutzung einfach und intuitiv ist. Hierbei sollten den Nutzenden ganz bewusst Entscheidungen erspart oder ermöglicht werden; was im UI- und UX-Design berücksichtigt sein kann.

12. Vertrauen ist ein wichtiger Bestandteil, der durch unterschiedliche Maßnahmen, die wir analysiert haben, geschaffen werden kann, z.B. viel Informationsaustausch der Transaktionspartner/innen und gegenseitige Bewertungen.

13. Die Nutzung von personenbezogenen Dienstleitungen über Online-Plattformen kann durch Berücksichtigung der genannten Punkte attraktiver sein als eine Beschäftigung in Schwarzarbeit.

Um diese Annahmen festigen und iterieren sowie mit zukünftiger Forschung unterstützen zu können, ist es essentiell, den Kunden von online gebuchten personenbezogenen Dienstleistungen, sowie deren Anbieter, noch besser zu verstehen und gegenüberzustellen. Nur so ist es möglich, Optimierungspotentiale zu erkennen und ein kundenzentriertes Angebot zu schaffen. Generell wurden Kundenbedürfnisse an onlinebuchbare personenbezogene Dienstleistungen, sowie das bestehende Angebot bisher wenig analysiert. Die momentan laufende Umfrage soll weitere und gefestigte Einblicke ermöglichen. Nur durch ein besseres Verständnis können Nutzungshürden herausgearbeitet und überwunden werden, sodass eine breite Nutzung und eine hohe Dienstleistungsqualität in Deutschland ermöglicht wird. Durch eine gesteigerte Nutzung ist ein wertvoller Beitrag zur deutschen Wirtschaft, zur Reduzierung des Fachkräftemangels und zur Steigerung der Verdienstmöglichkeiten geringqualifizierter Arbeitskräfte zu erwarten, wofür wir einen Beitrag mit unserer Forschung leisten.

Danksagung Dieser Beitrag ist Teil des vom Bundesministerium für Bildung und Forschung geförderten Verbundprojekts KUSTOMA mit dem Förderkennzeichen: 02K17A030, welches das Ziel hat, personenbezogene Dienstleistungen zu verbessern. Das Projekt konzentriert sich auf die Entwicklung einer Online- 
Plattform für personenbezogene Dienstleistungen. Herzlichen Dank gilt hier auch den Mitarbeitern der Verbundpartner sowie dem Projektträger Karlsruhe und den Studierenden der Forschungsgruppe „Uberize your life" der Technischen Universität München.

Funding Open Access funding provided by Projekt DEAL.

Open Access Dieser Artikel wird unter der Creative Commons Namensnennung 4.0 International Lizenz veröffentlicht, welche die Nutzung, Vervielfältigung, Bearbeitung, Verbreitung und Wiedergabe in jeglichem Medium und Format erlaubt, sofern Sie den/die ursprünglichen Autor(en) und die Quelle ordnungsgemäß nennen, einen Link zur Creative Commons Lizenz beifügen und angeben, ob Änderungen vorgenommen wurden.

Die in diesem Artikel enthaltenen Bilder und sonstiges Drittmaterial unterliegen ebenfalls der genannten Creative Commons Lizenz, sofern sich aus der Abbildungslegende nichts anderes ergibt. Sofern das betreffende Material nicht unter der genannten Creative Commons Lizenz steht und die betreffende Handlung nicht nach gesetzlichen Vorschriften erlaubt ist, ist für die oben aufgeführten Weiterverwendungen des Materials die Einwilligung des jeweiligen Rechteinhabers einzuholen.

Weitere Details zur Lizenz entnehmen Sie bitte der Lizenzinformation auf http://creativecommons.org/ licenses/by/4.0/deed.de.

\section{Literatur}

Becker C, Einhorn A, Grebe T (2012) Anbieter haushaltsnaher Dienstleistungen in Deutschland - Angebotsbedingungen, Strukturen, Perspektiven. Bundesministerium für Familie, Senioren, Frauen, und Jugend. https://www.bmfsfj.de/blob/93264/1a03566d76745fa7cb4e02ff97a212d9/anbieterhaushaltsnaher-dienstleistungen-in-deutschland-data.pdf. Zugegriffen: 15. März 2020

Bitkom e.V. (2015) Putzhilfe oder Kochservice aus dem Internet. Bitkom e. V. https://www.bitkom.org/ Presse/Presseinformation/Putzhilfe-oder-Kochservice-aus-dem-Internet.html. Zugegriffen: 15. März 2020

Bitkom e. V. (2017) Hilfe im Haushalt gesucht und online gebucht. Bitkom e. V. https://www.bitkom.org/ Presse/Presseinformation/Hilfe-im-Haushalt-gesucht-und-online-gebucht.html. Zugegriffen: 15. März 2020

Bonin H (2016) Haushaltsnahe Dienstleistungen als Instrument der Familienförderung. Konrad Adenauer Stiftung e.V. https://www.kas.de/documents/252038/253252/7_dokument_dok_pdf_47233_1.pdf/ 7eeead4e-2703-bad8-a8d9-c8cf309c9248?version=1.0\&t=1539649893741. Zugegriffen: 15. März 2020

Bundesministerium für Wirtschaft und Energie (2015) Haushaltsnahe Dienstleistungen - Qualitätsstandards als Chance für Wachstum und legale Beschäftigung. https://www.bmwi.de/Redaktion/DE/ Pressemitteilungen/2015/20150707-haushaltsnahe-dienstleistungen-qualitaetsstandards-chancewachstum-legale-beschaeftigung.html. Zugegriffen: 15. März 2020

Bundesministerium für Wirtschaft und Energie (2020) Dienstleistungen sichtbar gemacht: Zahlen und Trends auf einen Blick. https://www.bmwi.de/Redaktion/DE/Artikel/Mittelstand/ dienstleistungswirtschaft-01-zahlen-trends.html. Zugegriffen: 15. März 2020

Crößmann A, Mischke J, Hoffmann J (2018) Arbeitsmarkt auf einen Blick: Deutschland und Europa. Statistisches Bundesamt. https://www.destatis.de/Migration/DE/Publikationen/Thematisch/ Arbeitsmarkt/Erwerbstaetige/BroeschuereArbeitsmarktBlick.html?nn=217388. Zugegriffen: 15 . März 2020

Deutsche Bundesbank (2019) Vermögen und Finanzen privater Haushalte in Deutschland: Ergebnisse der Vermögensbefragung 2017. https://www.bundesbank.de/resource/blob/794130/d523cb34074622e1b4 cfa729f12a1276/mL/2019-04-vermoegensbefragung-data.pdf. Zugegriffen: 15. März 2020

Dickey MR (2017) IKEA has bought TaskRabbit. TechCrunch. https://techcrunch.com/2017/09/28/ikeabuys-taskrabbit/. Zugegriffen: 15. März 2020

Die Bundesregierung der Bundesrepublik Deutschland (2016) Bericht der Bundesregierung zur Lebensqualität in Deutschland. Presse- und Informationsamt der Bundesregierung. https://www. bmwi.de/Redaktion/DE/Publikationen/Wirtschaft/bericht-der-bundesregierung-zur-lebensqualitaetin-deutschland.pdf?_blob=publicationFile\&v=8. Zugegriffen: 15. März 2020 
Diener K, Götz S, Schreyer F, Stephan G, Lenhart J, Nisic N, Stöhr J (2015) Rückkehr ins Berufsleben nach familienbedingter Unterbrechung - Befunde der Evaluation der zweiten Förderperiode des ESFProgramms „Perspektive Wiedereinstieg“ des Bundesministeriums für Familie, Senioren, Frauen und Jugend. http://doku.iab.de/forschungsbericht/2015/fb0715.pdf. Zugegriffen: 15. März 2020 (Institut für Arbeitsmarkt- und Berufsforschung der Bundesagentur für Arbeit)

Eigenhüller L (2019) Service- und Beratungsstellen für Haushaltsnahe Dienstleistungen - Ein Modellprojekt zur Entwicklung des Arbeitsmarkts für haushaltsnahe Dienstleistungen. Institut für Arbeitsmarkt- und Berufsforschung der Bundesagentur für Arbeit. http://doku.iab.de/forschungsbericht/ 2019/fb0319.pdf. Zugegriffen: 15. März 2020

Enste DH, Heldman C (2017) Arbeitsplatz Privathaushalt - Minijobs und Schwarzarbeit von Haushaltshilfen. Institut der deutschen Wirtschaft Köln Akademie GmbH. https://www.iwkoeln.de/fileadmin/ publikationen/2017/361031/Gutachten_IW-Akademie_Arbeitsplatz_Privathaushalt_2017.pdf. Zugegriffen: 15. März 2020

Guerrero R, Lohrenz LS, Lattemann C, Robra-Bissantz S (2020) Digitalisierung (und Automatisierung) personennaher Dienstleistungen - eine bibliometrische Analyse. Automatisierung und Personalisierung von Dienstleistungen. Springer Gabler, Wiesbaden

Hawlitschek F, Teubner T, Weinhardt C (2016) Trust in the sharing economy. Unternehmung 70(1):26-44. https://doi.org/10.5771/0042-059X-2016-1-26

IfD Allensbach (2019) Anzahl der DINKS* (Double Income No Kids) in Deutschland von 2015 bis 2019. Statista. https://de.statista.com/statistik/daten/studie/264140/umfrage/dinks--anzahl-indeutschland/. Zugegriffen: 15. März 2020

IFOK GmbH, Institut der deutschen Wirtschaft Köln (2014) Professionalisierung haushaltsnaher Dienstleistungen durch Entwicklung und Etablierung von Qualitätsstandards. Bundesministerium für Wirtschaft und Energie. https://www.bmwi.de/Redaktion/DE/Publikationen/Studien/professionalisierunghaushaltsnaher-dienstleistungen-durch-entwicklung-und-etablierung-von-qualitaetsstandards.pdf? blob=publicationFile \&v=3. Zugegriffen: 15. März 2020

Ipsos (2013) Die Doppelverdienerfamilie ist das neue Ideal - mit Folgen Zukunftsforscher Opaschowski prophezeit Kulturwandel in Deutschland

Juncke D, Krämer L, Weinelt H (2019) Haushaltsnahe Dienstleistungen - Implementierung eines Fördermodells für haushaltsnahe Dienstleistungen. Prognos AG. https://www.prognos.com/uploads/tx_ atwpubdb/Endbericht_HHnaheDL_Prognos_090719.pdf. Zugegriffen: 15. März 2020

Kuckartz U (2016) Grundlagentexte Methoden. Qualitative Inhaltsanalyse. Methoden, Praxis, Computerunterstützung, Bd. 3. Beltz Juventa, Weinheim Basel

Lange G-M, Wodon Q, Carey K (2018) The changing wealth of nations 2018: building a sustainable future. The World Bank, Washington, DC. https://doi.org/10.1596/978-1-4648-1046-6

Lattemann C, Robra-Bissantz S, Fischer S, Ahmad R (2019) Personennahe Dienstleistungen in der digitalen Transformation. IM IO Fachmag 1(1):75-77

McKinsey \& Company (2016) Digitalisierung in deutschen Haushalten - Wie Familien mehr Qualitätszeit gewinnen können. https://www.bmfsfj.de/blob/100556/22337b52e49a0118ef08c6018584eece/ studie-digitalisierung-deutsche-haushalte-data.pdf. Zugegriffen: 15. März 2020

Nielen S, Kay R, Schröder C (2017) Disruptive Innovationen: Chancen und Risiken für den Mittelstand. Institut für Mittelstandsforschung Bonn. https://www.ifm-bonn.org//uploads/tx_ifmstudies/IfMMaterialien-259_2017.pdf. Zugegriffen: 15. März 2020

Presseportal (Hrsg) (2019) TaskRabbit startet heute in Deutschland. https://www.presseportal.de/pm/ 137345/4450488. Zugegriffen: 15. März 2020

Reinecke M, Gess C, Stegner K, Kröber R (2011) Machbarkeitsstudie „Haushaltsnahe Dienstleistungen für Wiedereinsteigerinnen“. Bundesministerium für Familie, Senioren, Frauen und Jugend. https://www. bmfsfj.de/blob/94264/84eb23b34594ee2c83b9db3d239d6908/machbarkeitsstudiehaushaltsnahedienstleistungen-fuer-wiedereinsteigerinnen-data.pdf. Zugegriffen: 15. März 2020

Robra-Bissantz S, Lattemann C, Guerrero R, Lux AM, Redlich B, Fischer S (2020) Der Mensch als Teil der Innovation - Eine ,Service Canvas“ als anwendungsorientierter Bezugsrahmen. Automatisierung und Personalisierung von Dienstleistungen. Springer Gabler, Wiesbaden

Steiner M, Steidle H, Matuschke M, Lehmann K, Schüssler R (2012) Dynamisierung des Marktes haushaltsnaher Dienstleistungen. Prognos AG. https://www.prognos.com/fileadmin/pdf/ publikationsdatenbank/121123_Prognos_Studie_Endbericht_Dynamisierung_Markt_HHDL.pdf. Zugegriffen: 15. März 2020

Thurmond VA (2001) The point of triangulation. J Nurs Scholarsh 33(3):253-258. https://doi.org/10.1111/ j.1547-5069.2001.00253.x 
Weber E, Zimmert F (2018) Arbeitszeiten zwischen Wunsch und Wirklichkeit: Wie Diskrepanzen entstehen und wie man sie auflöst. Institut für Arbeitsmarkt- und Berufsforschung der Bundesagentur für Arbeit. http://doku.iab.de/kurzber/2018/kb1318.pdf. Zugegriffen: 15. März 2020

Whillans AV, Dunn EW, Smeets P, Bekkers R, Norton MI (2017) Buying time promotes happiness. Proc Natl Acad Sci 114(32):8523-8527. https://doi.org/10.1073/pnas.1706541114

Wippermann C (2011) Haushaltsnahe Dienstleistungen: Bedarfe und Motive beim beruflichen Wiedereinstieg. Bundesministerium für Familie, Senioren, Frauen und Jugend. https://tu-dresden. de/karriere/ressourcen/dateien/berufung/dual_career_service_neuberufene/familie/haushaltsnahe_ dienstleistungen?lang=de. Zugegriffen: 15. März 2020 